\title{
FACTS2: A Service Oriented Simulation Framework for Aeronautical Communication System Evaluation
}

\author{
Thomas Gräupl \\ Institute of Communications and Navigation \\ Deutsches Zentrum für Luft- und Raumfahrt e.V. (DLR) \\ Oberpfaffenhofen, Germany \\ thomas.graeupl@dlr.de
}

\begin{abstract}
The modernization of the European air transportation system will increase the need for data communication. This creates the need for large-scale simulations to ascertain that new aeronautical communication systems fulfil the requirements of future air traffic management communication. This paper presents a framework for such simulations based on service-oriented software architecture. The Framework for Aeronautical Communications and Traffic Simulations 2 (FACTS2) is based on the concept of service oriented simulation creating complex simulations from simple software building blocks called "services". Separating the tasks of services appropriately allows for natural parallelization at the service level. FACTS2 was applied to the simulation of European reference air traffic for the years 2007 to 2035 that will provide the basis for future aeronautical communication evaluations. It could be shown that the service-oriented simulation approach offers significant performance gains through parallelization scaling linearly with the number of available CPUs. The air traffic simulation results were validated against published simulation results to verify their correctness.
\end{abstract}

Keywords - computer simulation; air traffic management; aeronautical communication; service oriented architecture.

\section{INTRODUCTION}

The EU and US programs for the sustainable modernization of the air transportation system - SESAR and NextGen - will change air traffic management significantly in the near future creating an increased need for efficient data communication with aircraft [1].

This large scale transformation requires also executing large scale air traffic management communication simulations to ascertain that future aeronautical communication systems meet the requirements of the envisioned performance oriented air traffic management concepts.

Various tools have been proposed to evaluate improved aeronautical communications systems. Khanna et al. [2] created the FASTE-CNS traffic analysis and capacity planning tool for communications. FASTE-CNS is implemented as a computation engine accessible through a web service. Kitaorwe et al. [3] used the OPNET simulator to compare the ACARS and VDL Mode 2 communication performance. Gomez and Ortiz [4] used $n s 2$ to evaluate CPDLC over VDL Mode 2. The $L$ band Digital Aeronautical Communication System (LDACS) was evaluated by Ayaz et al. [5] using OMNeT++ as described by Hoffmann et al. in [6].
It is a common feature of these simulators that they are barring basic model-view-controller separation - monolithic tools combining various tasks like air traffic generation, communication system simulation, and statistical analysis. Monolithic software designs are, however, inherently complex and difficult to maintain or modify [7].

This paper describes a simulation framework based on modern software architecture: Distributed large-scale air traffic management communication simulations organized in a parallelized toolchain of loosely coupled software services split by the separation of concerns. The service coupling is implemented such that it is suitable for parallel batch processing using the Portable Batch System (PBS) on high performance computers. The benefit of the approach is that it keeps the architectural complexity of the software low, and fosters the incorporation of existing simulation tools while promising significant performance gains through parallelization.

To demonstrate this new method we apply it to the simulation of reference air traffic for the evaluation of air traffic management communication systems. The simulation result is a data set of simulated reference air traffic for the year 2007 reflecting the observed number of IFR $^{1}$ flights in Europe [8], and a data set of simulated air traffic extrapolated for the years $2020,2025,2030$, and 2035 according to four growth scenarios published in the EUROCONTROL ${ }^{2}$ long term forecast [9]. The implementation of the simulation builds on the Future Aeronautical Communications Traffic Simulator (FACTS) by Hoffmann et al. [6] and is therefore called Framework for Aeronautical Communications and Traffic Simulations 2 (FACTS2).

\section{DEFINITIONS}

In this paper we follow Hürsch and Lopes [7] in the definition of separation of concerns: Separation of concerns separates the basic algorithm from special purpose concerns, allowing the locality of different kinds of information in the programs, making them easier to write, understand, reuse, and modify.

\footnotetext{
${ }^{1}$ Flights conducted according to Instrument Flight Rules (IFR) navigate by electronic signals independently of visibility.

${ }^{2}$ EUROCONTROL is the European organization for the safety of air navigation.
} 
In the definition of service oriented computing we follow Papazoglou [10]: Service-oriented computing is the computing paradigm that utilizes services as fundamental elements for developing applications/solutions. Services are self-describing, platform-agnostics computational elements that support rapid, low-cost composition of distributed applications.

We define service oriented architectures according to the same author [10]: Service oriented architecture is a way of reorganizing software applications and infrastructure into a set of interacting services.

\section{METHODS}

The simulation method presented in this paper is based on the concept of service oriented simulation [11], which is the application of service oriented computing to computer simulation problems.

The notion of service oriented computing is based on the loose coupling of services; each service contributing its specific capabilities to create an overall solution. Services can be viewed as producers, offering locally available resources or abilities to their consumers through well-known interfaces. A benefits chain leading to the ultimate solution is being built by the information flow from service to service independently from the underlying implementation. Thus, each consumer may utilize any collection of services to create the required solution.

The application of service oriented computing to simulation problems results in a service oriented simulation architecture based on the interaction of self-contained simulation services. This results in a number of benefits: Due to the distribution of the workload each service or tool has to deal with one particular aspect of the simulation only, making the overall solution less constraint by computational limitations and thus more scalable; parallelization is often easily achievable on service level. The decomposition of the simulation problem into selfcontained services results in less complex software units split by the separation of concerns that are easier to develop and maintain. The flexible combination of existing services enables the quick formulation of new solutions reusing existing implementations.

The service interface is of major concern in service oriented computing. Conventional services exchange XML encoded data over the network to achieve location transparency [10].

Contrary to conventional service oriented architectures the service oriented simulation approach of this paper does not use a network transparent interface as this is problematic in supercomputing environments. The simulation uses compressed XML encoded files as interface. This means that the service oriented simulation approach does not fulfil all requirements of [10]: The services are technology-neutral and loosely coupled, however, the absence of location transparency implies that the service oriented simulation approach presented in this paper does not qualify as service oriented architecture as defined by Papazoglou.

The simulation problem addressed in this paper is the generation of reference air traffic intended as input for further simulations evaluating existing and future air traffic management communication systems. The simulation result shall comprise

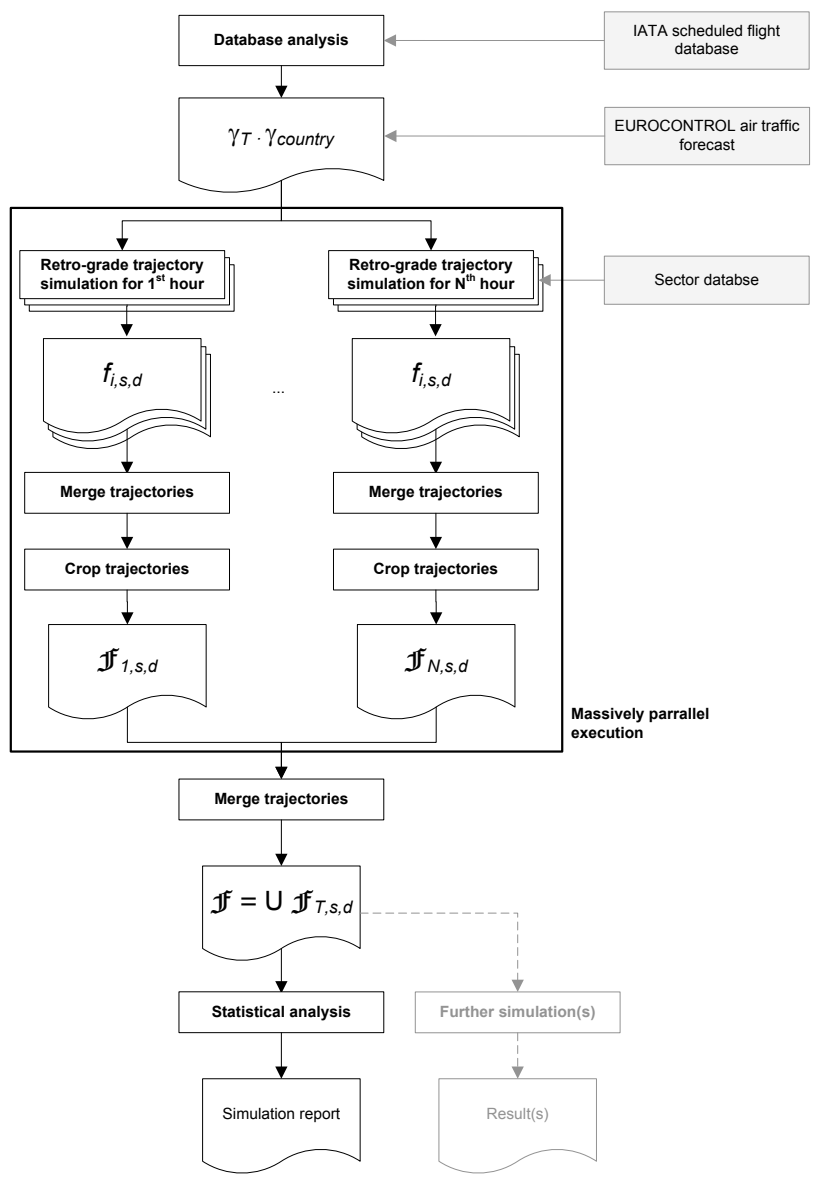

Fig. 1. Service interaction for the simulation of reference air traffic: The simulation starts with the analysis of the IATA database of scheduled IFR flights. Individual flight trajectories are then simulated according to the frequency and expected growth forecast for the next two decades. The individual trajectories are combined and cropped to a reference day. This reference day is then statistically analyzed or used as inpurt for further simulations.

simulated air traffic reflecting the number of IFR flights reported for the years 2007, and the number of IFR flights expected for 2020 to 2035 for different growth scenarios in Europe.

The air traffic simulation is implemented as a service oriented simulation of five interacting services: Scheduled flight database analysis, trajectory simulation, trajectory merging, trajectory cropping, and statistical analysis of the final simulation result. The interaction of the simulation services is illustrated in Fig. 1 and discussed in detail below.

The services are invoked by the Portable Batch System (PBS). PBS is a computer software for job scheduling on Unix computing grids. It takes care of interdependencies between services and tries to utilize the hardware as efficiently as possible by the parallel invocation of service instances. 


\section{A. Database Analysis}

The first service in the overall simulation is the analysis of the IATA $^{3}$ database of worldwide scheduled passenger and cargo IFR flights for the years 2007 and 2008. This service was implemented by Hoffmann et al. as reported in [12] and we reuse Hoffmann's implementation and results.

The database analysis restricts itself to two reference days: May $21^{\text {st }}-22^{\text {nd }}, 2007$. Hoffmann identified these days as typical days exhibiting an average number of daily flights. The database entries for these days were analyzed to create a statistical model of average European air traffic patterns. The flight patterns of the second day define the actual reference day for 2007. The flight patterns of the first day were included in the analysis to take over-night flights into account.

For each hour of the reference day Hoffmann used the number of on-going flights between any two European airports as basis for the calculation of the hourly aircraft generation rate $\gamma_{T}(s, d)$ for each pair of source and destination airports $s$ and $d$, where $s, d \in \mathfrak{A}$, and $\mathfrak{A}$ is the set of airports in the flight plan database. $T \in\left\{T_{0}, \ldots, T_{23}\right\}$ represents the 24 hours of the reference day.

The area of interest for the simulation is the region between $35^{\circ} \mathrm{N} 60^{\circ} \mathrm{N}$ and $10^{\circ} \mathrm{W} 30^{\circ} \mathrm{E}$, which covers continental Europe. Along the boundary of this spherical rectangle, entry and exit points were created for flights entering or leaving European airspace. Inter-continental flights use therefore one such point in lieu of a source or destination airport.

\section{B. Retro-Grade Trajectory Simulation}

On the basis of Hoffmann's 2007 flight generation rates we simulated a reference day of average European air traffic for the years 2007, and four forecast scenarios for the years 2020 to 2035 , each.

Flights are simulated individually. It is therefore possible to perform these simulations in parallel to speed up the overall simulation. The simulation of each flight trajectory is implemented as a time-stepped simulation.

Each flight is represented by a function $f(t)$ returning its state vector as a function of time

$$
f(t)=\mathfrak{s}_{t} \text { for } \alpha \leq t \leq \omega
$$

where $\mathfrak{s}_{t}$ is the state vector of the flight $f$ at time $t . \alpha$ is the start time of the flight, $\omega$ is the end time of the flight. $\mathfrak{s}_{t}$ comprises the position, altitude, sector, and domain of the flight and is calculated as described below.

Flight trajectories are simulated in reverse (retro-grade) time order, because the intended follow-up simulations of air traffic management communication systems can be simplified when information on future events is available e.g. the time a flight will remain in its current sector or domain. To this end we simulate individual flight trajectories with $\omega \geq t \rightarrow \alpha$

${ }^{3}$ The International Air Transport Association (IATA) is a trade association of the world's airlines.

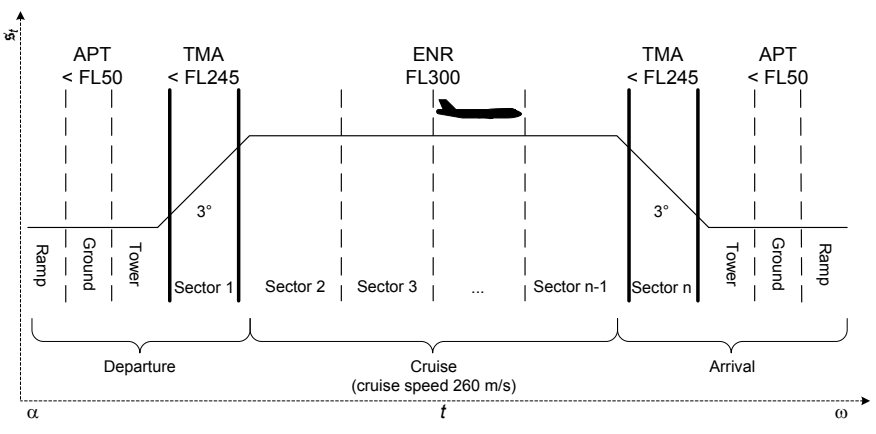

Fig. 2. Illustration of the flight state vector $\mathfrak{s}_{t}$ as function of time $t$. The position component of the state vector $\mathfrak{s}_{t}$ is not displayed.

where $\mathrm{t}$ converges to $\alpha$ from above. Note that the simulation result is, however, provided in normal (ante-grade) time order at the service interface.

The current Air Traffic Control (ATC) sector of a flight is inferred from its current position and an ATC sector database obtained by the German Aerospace Center (DLR).

In the spatial domain all flights follow great-circle routes from departure to destination airport and have simplified trapezoidal altitude profiles. The combination of great-circle routes and simplified altitude profiles is deemed to provide sufficient fidelity for the intended simulation of large scale communication systems.

The flight domain is calculated from the current altitude ${ }^{4}$ of the flight. Flights below FL50 5 are considered to be in the airport domain (APT). Flights below FL245 are in the Terminal Maneuvering Area (TMA), and flights above this threshold are in the en-route (ENR) domain. The only deviation from this mechanism is applied when the flight is not within any ATC sector. In this case the domain is assumed to be Oceanic, Remote, or Polar (ORP).

In the APT domain it is assumed that the flight enters the simulation 30 minutes before takeoff. After landing the flight remains additional 15 minutes in the simulation at the airport.

All other parameters of the flight are uniformly randomly distributed $+/-10 \%$ around a configurable average value: Climb/descent angle $\left(3^{\circ}\right)$, cruise speed $(260 \mathrm{~m} / \mathrm{s})$, and cruise altitude (FL300). As of now, the cruise speed is assumed to be constant from take-off to landing.

An illustration of the flight state vector $\mathfrak{s}_{t}$ of as a function of time $t$ is displayed in Fig. 2.

The simulation of the flight trajectories builds on the work of Hoffmann, Khanna, and Rokitansky [13][14]. All three simulators assume great circle routes with varying pre-flight and post-flight times at the airport. Rokitansky uses a $1^{\circ} \times 1^{\circ}$ geographical degree grid for TMA and $5^{\circ} \times 5^{\circ}$ geographical degree grid for ENR instead of an ATC sector database. A comparison table of the simulation fidelity is provided in TABLE I.

\footnotetext{
${ }^{4}$ These altitude levels correspond to the altitude levels assumed in [17] .

51 Flight Leve (FL) $=100 \mathrm{ft}$ altitude.
} 
TABLE I. COMPARISON OF SIMULATION FIDELITY

\begin{tabular}{rcccc}
\hline & FACTS2 & $\begin{array}{c}\text { Hoffmann } \\
{[6]}\end{array}$ & $\begin{array}{c}\text { Khanna } \\
{[2]}\end{array}$ & $\begin{array}{c}\text { Rokitansky } \\
{[15]}\end{array}$ \\
\hline Great circle routes & + & + & + & + \\
Take-off time at airport & $30 \mathrm{~min}$ & - & $15 \mathrm{~min}^{6}$ & $30 \mathrm{~min}$ \\
Landing time at airport & $15 \mathrm{~min}$ & - & $25 \mathrm{~min}$ & $15 \mathrm{~min}$ \\
Flight speed & $260 \mathrm{~m} / \mathrm{s}$ & $260 \mathrm{~m} / \mathrm{s}$ & variable & variable \\
$\begin{array}{r}\text { Flight domain according to } \\
\text { current altitude }\end{array}$ & + & + & - & + \\
ATC sectors & database & database & - & TMA: $1^{\circ} \times 1^{\circ}$ \\
ENR: $5^{\circ} \times 5^{\circ}$ \\
\hline
\end{tabular}

\section{Air Traffic Growth}

The IATA database available lists only the scheduled passenger and cargo IFR flights of 2007 and 2008. In order to obtain air traffic scenarios for later years the number of IFR flights has thus to be extrapolated.

The number of IFR flights is extrapolated by applying growth factors to the air traffic generation rates $\gamma_{T}$. EUROCONTROL published per-country growth factors in [8] fig. 29 and [9] fig. 38. These growth factors were applied to all flights departing from the same country. That is, we let

$$
\gamma_{T}(s, d) \leftarrow \gamma_{c, y} \cdot \gamma_{T}(s, d)
$$

in (4) and (5) below where $\gamma_{c, y}$ is the per-country growth factor for the year $y$, and $s$ is an airport in country $c . \gamma_{c, y}$ is calculated from the ratio of forecast number of IFR flights $I_{c}$ for country $c$ and the year of interest $y$ to the reference year 2007

$$
\gamma_{c, y}=\frac{I_{c}(y)}{I_{c}(2007)}
$$

for each country in the area of interest. Note that EURCONTROL published forecast numbers for scheduled IFR flights for four growth scenarios for the years 2020 to 2035: Scenario A (highest growth), Scenario C, Scenario C', and Scenario D (lowest growth).

The growth factor for IFR flights arriving and departing from European air space [9] fig. 34 was applied to flights entering from outside of Europe in a similar way to the per-country growth factors.

\section{Trajectory Merging}

The flight trajectories are simulated individually and have thus to be merged. For each hour of the reference day individual flights $f_{i}$ are generated such that the time between flight start times $\alpha_{i}$ is exponentially distributed according to the flight generation rate extracted from the flight plan database. The flight generation rate is $1 /$ (inter-arrival time) according to Hoffmann et al. [6]. Flights $f_{i}$ are thus generated with exponentially distributed inter-arrival times such that $\alpha_{i+1}-\alpha_{i} \sim$ $\operatorname{Exp}\left(\gamma_{T}(s, d)\right)$ for all $s, d \in \mathfrak{A}$.

The set of flights $\mathfrak{F}_{T, s, d}$ that depart within hour $T$ of the reference day from airport $s$ to airport $d$ is thus

\footnotetext{
${ }^{6}$ Each aircraft is simulated as a "typical" flight (take-off 15 mins, en-route 65 minutes, landing 25 mins).
}

$$
\begin{aligned}
\mathfrak{F}_{T, s, d}=\left\{f_{i, s, d}(t) \mid \alpha_{i+1}-\alpha_{i}\right. \\
\left.\sim \operatorname{Exp}\left(\gamma_{T}(s, d)\right) \text { and } \alpha_{i} \in T\right\}
\end{aligned}
$$

with $s, d \in \mathfrak{A}$ and $T \in\left\{T_{0}, \ldots, T_{23}\right\}$. Note that flights need not end during the given time period $T$, that is they may continue on the day after the reference day.

Some flights starting on the day before the reference day continue into the reference day and have thus to be taken into account. The set of flights of the last six hours of the day before the reference day merged with the flights of the reference day is

$$
\begin{gathered}
\mathfrak{\mho}_{30 h}=\bigcup_{T, s, d} \mathfrak{F}_{T, s, d}= \\
\bigcup_{T, s, d}\left\{f_{i, s, d}(t) \mid \alpha_{i+1}-\alpha_{i} \sim \operatorname{Exp}\left(\gamma_{T}(s, d)\right), \alpha_{i} \in T\right\}
\end{gathered}
$$

for $T=T_{-6}, \ldots, T_{0}, \ldots, T_{23}$ and $\forall s, d \in \mathfrak{A}$. No flights of $T_{-6}$ or earlier ended after midnight.

Note that all operations up to now, but the union operation, can be performed in parallel.

\section{E. Trajectory Cropping}

So far the simulated flights extend into the last hours of the day before the reference day and the first hours of the day after the reference day.

Cropping the flight trajectories to the reference day we get the set of state vectors of the flights of the reference day $\mathfrak{F}(t)$ as function of time

$$
\mathfrak{F}(t)=\left\{f(t) \mid f \in \mathfrak{F}_{30 h} \text { and } t \in\left\{T_{0}, \ldots, T_{23}\right\}\right\}
$$

\section{F. Statistical Analysis}

The result of the air traffic simulation is stored in an XML file listing the state vectors of all simulated flights $\mathfrak{F}(t)$ of the reference day with a time resolution of $\Delta t=60 \mathrm{~s}$.

The simulation result is then analyzed for two statistics to evaluate the quality of the result: $|\mathfrak{F}|$ is the number of IFR flights on the reference day; $\max _{t}|\mathfrak{F}(t)|$ is the maximum number of concurrent flights at the same time, which is also known as the Peak Instantaneous Aircraft Count (PIAC) of the reference day.

\section{RESULTS}

The simulation yields 18 reference days of air traffic as result: One reference day for 2007 and 2012 each, and four extrapolated scenarios for 2020, 2025, 2030, and 2035, each.

Fig. 3 and Fig. 4 illustrate an example trajectory of a flight from Oberpfaffenhofen airport (EDMO) to Prague airport (LKPR) in terms of position, altitude, and ATC sectors.

\section{A. Reference air traffic 2007}

The PIAC is $\max _{t}|\mathfrak{F}(t)|=2,983$ for the 2007 reference day. The PIAC is plotted with $1^{\circ} \times 1^{\circ}$ degree geographic reso- 


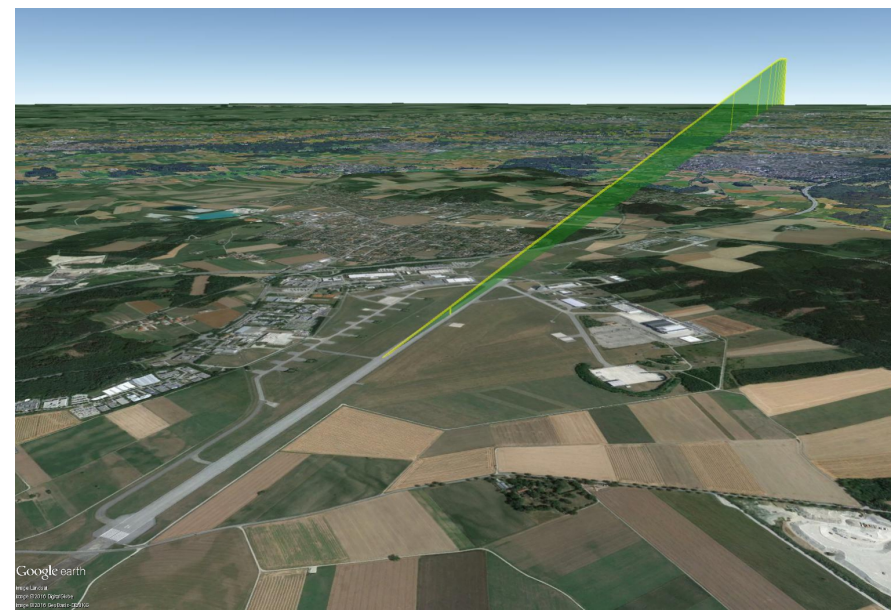

Fig. 3. Flight from EDMO to LKPR; latitude, longitude, and altitude component of $f(t)=s_{t}$ at take-off. Image: Google Earth.

lution in Fig. 5. Note that the PIAC is calculated for each $1^{\circ} \times$ $1^{\circ}$ degree square separately in this map.

The number of simulated flights of the 2007 reference day is $|\mathfrak{F}|=26,952$. The graph of the number of flights $|\mathfrak{F}(t)|$ as a function of time is shown in Fig. 6.

\section{B. Extrapolated Air Traffic 2012 to 2035}

The application of the air traffic growth factors gained from [8] and [9] results in extrapolated scenarios for the years 2020 to 2035 . The number of flights $|\mathfrak{F}|$ in each scenario is shown in TABLE II.

\section{Computation Time}

The computation time ${ }^{7}$ for the 2007 reference day is approximately 63 minutes without parallelization and approximately 17 minutes with parallelization over four CPUs providing eight threads with Hyper-Threading. This corresponds to an approximated speed-up of 3.7 that may be improved by increasing the number of CPUs.

\section{DISCUSSION}

The presented framework is suitable to implement large scale simulations with low architectural complexity and the opportunity for performance gains through parallelization. It yields large result data sets -18 days of air traffic - with sufficient simulation fidelity for communication system evaluations, and with relatively short computation times.

The air traffic simulation result for the 2007 reference day can be validated against the PIAC results published by Rokitansky et al. in [15]. Rokitansky uses an independent flight database as basis for his simulations. The document [16] reports the use of $\mathrm{OAG}^{8}$ world-wide scheduled flight plan data (March $19^{\text {th }} 2007$ - March $18^{\text {th }} 2008$ ) and EUROCONTROL $\mathrm{CFMU}^{9}$ data for Aug. $31^{\text {st }}$ 2007. Further, it is noted that the

\footnotetext{
${ }^{7}$ Intel Xeon at $4 \times 3.6 \mathrm{GHz}, 32 \mathrm{~GB}$ RAM, SSD.

${ }^{8}$ OAG (oag.com) is an air travel intelligence company.

${ }^{9}$ The Central Flow Management Unit (CFMU) coordinates all European flights.
}

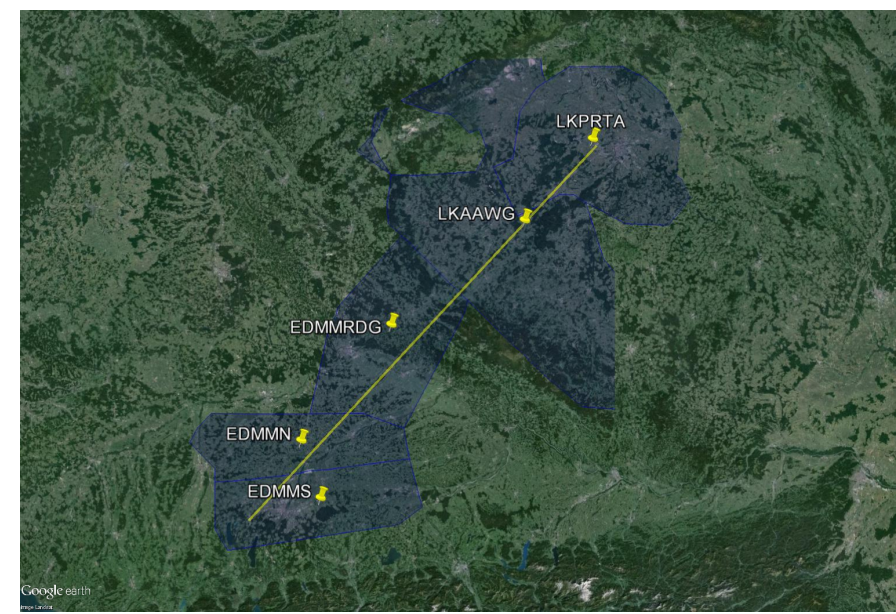

Fig. 4. Flight from EDMO to LKPR; great-circle route with passed sectors highlighted; latitude, longitude (yellow), and current sector (blue, with pins) component of $f(t)=s_{t}$. Image: Google Earth.

number of flights recorded by CFMU is greater than the number of scheduled flights. Rokitansky therefore applies an empirical correction (growth) factor of 1.4 to account for the flights missing in the database e.g. short-term business flights, military missions, general aviation, and the like.

When the same correction factor is applied to the FACTS2 simulations our methods yields comparable results as shown in TABLE III. We claim therefore that the FACTS2 results, considering the correction factor for non-IFR flights, do not conflict with Rokitansky's results, but capture the typical European scheduled IFR air traffic as was the objective of the simulation. This claim is further supported by EUROCONTROL results discussed below. It should be noted that our results cannot be validated against Rokitansky's results for other years than 2007, as he uses an older version of the EUROCONTROL air traffic forecast.

The FACTS2 results can also be validated against the recorded and forecast number of European IFR flights published by the EUROCONTROL Statistics and Forecast Service (STATFOR) in [8] and [9]. Both documents give only the number of scheduled IFR flights per year. The average number of scheduled IFR flights per day is therefore calculated by dividing the yearly number by 365 . The average recorded and forecast number of daily scheduled IFR flights in Europe is summarized in TABLE IV.

The deviation of the FACTS2 results from the STATFOR values is shown in TABLE V. It indicates that our results correspond very well with the EUROCONTROL forecast. The deviation of the FACTS2 results from the forecast is always less than $3.04 \%$ and mostly below $1.0 \%$.

The measured computation times indicate that the parallelization speed-up scales approximately linearly with the number of CPUs available. This result was to be expected taking the design of the service interaction in Fig. 1 into account. The parallelization speed-up is - for the same reason - limited by the maximum number of services that can run in parallel. In the problem at hand the upper limit of the speed-up is thus 30 for the 30 hours of air traffic simulated. 
Future work will use FACTS2 and the air traffic simulation results of this paper to simulate the data traffic load generated by future air traffic management applications. The combined results will then provide the basis to evaluate new air traffic management communication systems in large-scale performance simulations.

\section{CONCLUSION}

In this work we presented a simulation framework for large-scale air traffic management communication simulations that is based on modern software architecture.

The service oriented simulation approach applied in FACTS2 allows creating complex simulations from simple building blocks called "services" that can be reused for other simulation tasks. Dividing the overall simulation problem into services according to the principle of "separation of concerns" yields simpler and smaller units of software that are easier to modify and maintain.

The presented approach has been applied to the simulation of 18 days of European air traffic. It has been shown that FACTS2 offers significant performance gains through parallelization and that the results can be validated against the published literature.

TABLE II. FACTS2: SIMULATED NUMBER OF IFR FLIGHTS PER DAY

\begin{tabular}{rllllll}
\hline $\begin{array}{r}\text { FACTS2 } \\
\text { (IFR flights) }\end{array}$ & 2007 & 2012 & 2020 & 2025 & 2030 & \multirow{2}{*}{2035} \\
\hline Observed growth & 27,219 & 26,952 & & & & \\
Scenario A & & & 34,088 & 38,617 & 42,430 & 46,663 \\
Scenario C & & & 31,385 & 34,668 & 37,312 & 39,250 \\
Scenario C' & & & 31,185 & 33,541 & 35,581 & 37,392 \\
Scenario D & & 28,533 & 29,673 & 30,143 & 30,951 \\
\hline
\end{tabular}

TABLE III. COMPARISON OF FACTS2 AND ROKITANSKY [13] PIAC RESULTS FOR THE REFERENCE DAY 2007

\begin{tabular}{rccc}
\hline $\begin{array}{r}\text { PIAC } \\
\text { (IFR flights) }\end{array}$ & FACTS2 & FACTS2 & Rokitansky [15] \\
\hline Correction factor for flight gen. rate & 1 & 1.4 & 1.4 \\
PIAC 2007 & 2,983 & 3,894 & $3,995^{10}$ \\
\hline
\end{tabular}

TABLE IV. EUROCONTROL FORECAST OF AVERAGE NUMBER OF IFR FLIGHTS PER DAY ACCORDING TO [8] FOR 2007 AND [9] FOR 2012 TO 2035

\begin{tabular}{rcccccc}
\hline $\begin{array}{r}\text { EUROCONTROL } \\
\text { (IFR flights) }\end{array}$ & 2007 & 2012 & 2020 & 2025 & 2030 & 2035 \\
\hline Observed growth & 27,515 & 26,158 & & & & \\
Scenario A & & & 34,205 & 38,737 & 43,148 & 47,501 \\
Scenario C & & & 31,263 & 34,414 & 37,041 & 39,332 \\
Scenario C' & & & 31,063 & 33,523 & 35,658 & 37,723 \\
Scenario D & & & 27,929 & 29,074 & 29,699 & 30,819 \\
\hline
\end{tabular}

TABLE V. DEVIATION OF FACTS2 SIMULATION RESULT FROM EUROCONTROL FORECAST

\begin{tabular}{rrrrrrr}
\hline FACTS2 / EUROCONTROL & 2007 & 2012 & 2020 & 2025 & 2030 & 2035 \\
\hline \%) & 208 & -3.04 & & & & \\
Observed growth & 1.08 & & 0.34 & 0.31 & 1.66 & 1.76 \\
Scenario A & & & -0.39 & -0.74 & -0.73 & 0.21 \\
Scenario C & & & -0.39 & -0.05 & 0.22 & 0.88 \\
Scenario C' & & & -2.16 & -2.06 & -1.49 & -0.43 \\
Scenario D & & &
\end{tabular}

${ }^{10}$ PIAC for the FACTS2 area of interest calculated from the [16] dataset.

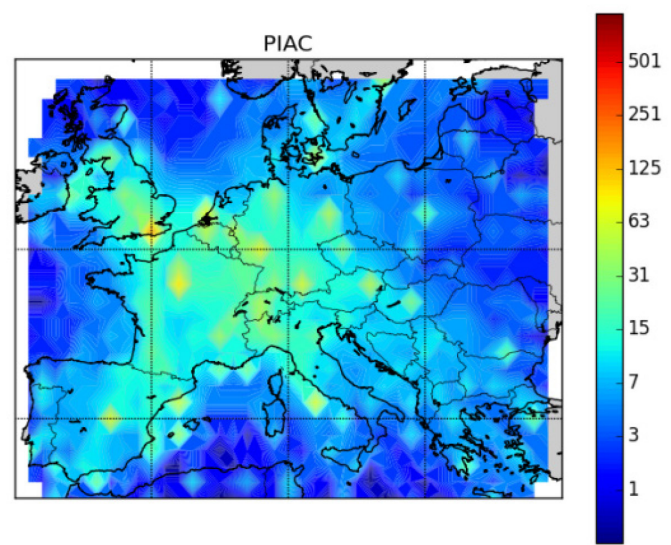

Deutsches Zentrum fuer Luft- und Raumfahrt www.dlr.de

Fig. 5. PIAC $=\max _{t}|\mathfrak{F}(t)|$ with a geographic resolution of $1^{\circ} \times 1^{\circ}$ degree for the area of interest.

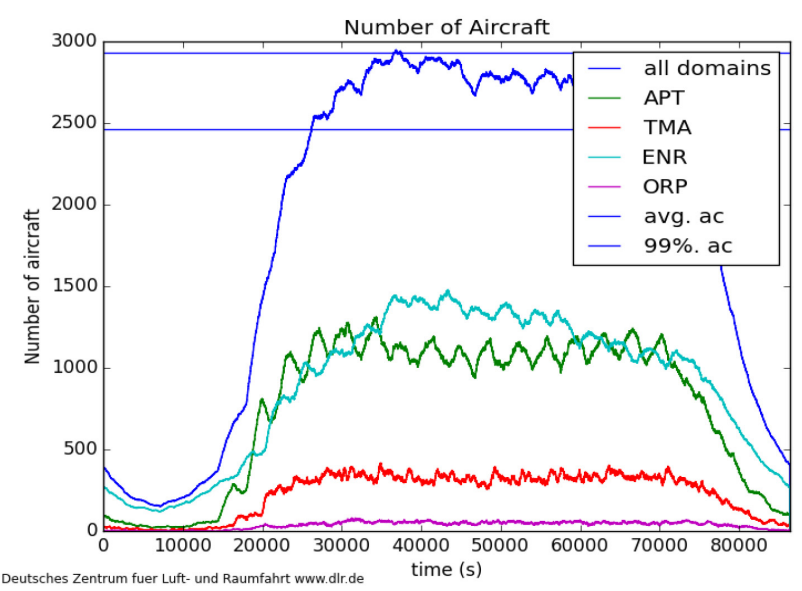

Fig. 6. Number of aircraft $|F(t)|$ in the area of interest; total, and for each domain separately.

\section{REFERENCES}

J. P. McGee, R. Parasuraman, A. S. Mavor, C. D. Wickens, and others, The Future of Air Traffic Control:: Human Operators and Automation. National Academies Press, 1998.

[2] M. Khanna, C. Dhas, C. Wargo, S. Vidyanandan, M. Joseph, C. Netto, and K. Wargo, "Results of field trials and features of FASTECNS-a traffic analysis and capacity planning tool for communications, navigation and surveillance," in Digital Avionics Systems Conference, 2003. DASC'03. The 22nd, 2003, vol. 1, p. 4 B.

[3] J. Kitaori, "A performance comparison between VDL mode 2 and VHF ACARS by protocol simulator," in Digital Avionics Systems Conference, 2009. DASC'09. IEEE/AIAA 28th, 2009, p. 4-B.

L. Gomez and J. Ortiz, "Modeling and simulation of VDL mode 2 subnet for CPDLC in El Dorado airport," in Digital Avionics Systems Conference (DASC), 2013 IEEE/AIAA 32nd, 2013, pp. 3B4-1.

S. Ayaz, F. Hoffmann, R. German, and F. Dressler, "Analysis of deficit round robin scheduling for future aeronautical data link," in 
IEEE International Symposium on Personal, Indoor and Mobile Radio Communications, PIMRC, 2011, pp. 1809-1814.

[6] F. Hoffmann, C. Bauer, D. Medina, and S. Ayaz, "FACTS: An $\mathrm{OMNeT}++$ Based Simulator for Aeronautical Communications," in Proceedings of the 1st International Conference on Simulation Tools and Techniques for Communications, Networks and Systems \& Workshops, 2008, pp. 85:1-85:4.

[7] W. L. Hursch and C. V. Lopes, "Separation of Concerns," Computer (Long. Beach. Calif)., pp. 1-20, 1995.

[8] EUROCONTROL, "Long-Term Forecast: IFR Flight Movements 2010-2030," 2010. [Online]. Available: http://www.eurocontrol.int/articles/challenges-growth.

[9] EUROCONTROL, "Challenges of Growth 2013 - Task 4: European Air Traffic in 2035," 2013. [Online]. Available: http://www.eurocontrol.int/articles/challenges-growth.

[10] M. P. Papazoglou, "Service-oriented computing: concepts, characteristics and directions," in Web Information Systems Engineering, 2003. WISE 2003. Proceedings of the Fourth International Conference on, 2003, pp. 3-12.

[11] M. Ehammer, T. Graeupl, and C. H. Rokitansky, "Applying SOA concepts to the simulation of aeronautical wireless communication," in Proceedings of the 11th communications and networking simulation symposium, 2008, pp. 194-201.
$[12]$

F. Hoffmann, U. Epple, M. Schnell, and U. Fiebig, "Feasibility of LDACS1 cell planning in European airspace," in Digital Avionics Systems Conference (DASC), 2012 IEEE/AIAA 31st, 2012, pp. 5E11-5E1-13.

[13] C.-H. Rokitansky, M. Ehammer, and T. Gräupl, "NEWSKY--Novel simulation concepts for future air traffic," Proc. 1st CEAS Eur. Air Sp. Conf., pp. 611-618, 2007.

[14] C.-H. Rokitansky, M. Ehammer, and T. Gräupl, "NEWSKY Building a simulation environment for an integrated aeronautical network architecture," in AIAA/IEEE Digital Avionics Systems Conference - Proceedings, 2007, pp. 4B41-4B411.

[15] C.-H. Rokitansky, M. Ehammer, and T. Gräupl, "Communication capacity assesment for the Iris satellite system," in Digital Avionics Systems Conference, 2008. DASC 2008. IEEE/AIAA 27th, 2008, p. 2-B.

[16] C.-H. Rokitansky, W. Brandner, M. Ehammer, T. Gräupl, M Messner, and A. Zimmerebner, "PIAC Analysis Report," 2008. [Online]. Available: https://artes.esa.int/projects/technicalassistance-iris-communication-standards-evaluation-universitysalzburg.

[17] EUROCONTROL and FAA, "Communication Operating Concept and Requirements for the Future Radio System, Ver. 2.0,” 2007. 